\title{
A cis-to-trans switch in DTD-like fold relaxes substrate chiral specificity
}

Santosh Kumar Kuncha ${ }^{1}$, Mohd Mazeed ${ }^{1}$, Raghvendra singh ${ }^{1}$, Bhavita Kattula ${ }^{1}$, Sambhavi pottabathini ${ }^{1}$, Rajan Sankaranarayanan ${ }^{1}$

${ }^{1}$ CSIR-Centre For Cellular And Molecular Biology, Hyderabad, India E-mail: santoshkumar@ccmb.res.in

D-aminoacyl-tRNA deacylase (DTD)-like fold is a primodial fold which is present in all three domains of life and is involved in proofreading during translation of the genetic code. DTD-like fold in archaea is present as an N-terminal domain (NTD) of threonyl-tRNA synthetase (ThrRS) to remove L-serine that is mischarged on tRNAThr by the ThrRS aminoacylation domain [1]. In bacteria and eukarya, DTD-like fold is present as a free-standing proofreading domain named D-aminoacyl-tRNA deacylase. DTD has been implicated in maintaining homochirality of the proteome by specifically scavenging and decoupling any D-amino acid attached to a tRNA, thereby excluding D-amino acids from the translational machinery. This absolute "DTD's enantioselectivity or stereospecificity" is achieved by an invariant cross-subunit Gly-cisPro motif (GP motif), which helps to reject even the smallest L-amino acid (i.e., L-Ala), thereby allowing only D-amino acids or achiral glycine to occupy the DTD catalytic site $[2,3]$. The GP motif of DTD is rigidly fixed by surrounding residues through multiple main chain interactions; a highly conserved DTD-specific arginine (R7) anchors the GP motif through its side chain. On analysis of structure-based sequence alignment of all the available DTD sequences, we could find numerous sequences which lack R7 and have less than $25 \%$ sequence identity. This variant of DTD (which we have named XDF) is present in kingdom animalia in addition to DTD. We have solved a high-resolution crystal structure of XDF from mouse at $1.86 \AA$ resolution. Interestingly, the structure reveals that the GP motif of XDF is in trans conformation with its carbonyl oxygens oriented parallel and pointing inwards away from the active site (i.e., towards the protein core). This is in contrast to DTD's GP motif, wherein the carbonyl oxygens are oriented parallel and protruding outwards into the active site (i.e., away from the protein core). The $180^{\circ} \mathrm{GP}$ motif flip due a cis-to-trans switch in XDF has led to a gain of function and relaxation of substrate chiral specificity, i.e., unlike DTD, XDF acts on L-aminoacyl-tRNAs in addition to D-aminoacyl-tRNAs. Overall, we show how a cis-to-trans switch in an animalia-specific DTD variant has increased the repertoire of substrates for DTD-like fold. The physiological relevance of this evolutionary gain of function is currently being probed

[1] Ahmed, S. et al. (2015). Nat. Commun. 6:7552, 1-12

[2] Ahmad, S., Routh, S. B. et al. (2013). Elife, 2:e01519, 1-18.

[3] Routh, S. B. et al. (2016). PLoS Biol. 14:e1002465, 1-22.

Keywords: Translation, genetic code, proofreading/editing 